\title{
$p$-Stability and $p$-Stabilizability of Stochastic Nonlinear and Bilinear Hybrid Systems under Stabilizing Switching Rules
}

\author{
Ewelina Seroka and Lesław Socha \\ Faculty of Mathematics and Natural Sciences, College of Sciences, Cardinal Stefan Wyszyński University in Warsaw, \\ Dewajtis Street 5, 01-815 Warsaw, Poland
}

Correspondence should be addressed to Ewelina Seroka; ewelina.seroka@gmail.com

Received 9 November 2012; Accepted 14 January 2013

Academic Editor: Piyapong Niamsup

Copyright ( 2013 E. Seroka and L. Socha. This is an open access article distributed under the Creative Commons Attribution License, which permits unrestricted use, distribution, and reproduction in any medium, provided the original work is properly cited.

\begin{abstract}
The problem of $p$ th mean exponential stability and stabilizability of a class of stochastic nonlinear and bilinear hybrid systems with unstable and stable subsystems is considered. Sufficient conditions for the $p$ th mean exponential stability and stabilizability under a feedback control and stabilizing switching rules are derived. A method for the construction of stabilizing switching rules based on the Lyapunov technique and the knowledge of regions of decreasing the Lyapunov functions for subsystems is given. Two cases, including single Lyapunov function and a a single Lyapunov-like function, are discussed. Obtained results are illustrated by examples.
\end{abstract}

\section{Introduction}

The problem of stability and stabilization of dynamic systems is one of the basic problems in the control theory. It is well known that there are classes of control systems which cannot be stabilized by a single feedback control [1]. In this case and in the case of the hybrid control systems, switched controls can assure the stability.

Liberzon and Morse in [2] mention that one of the basic problems for dynamic systems is the construction of stabilizing switching laws [1,3-6]. It is known that if a common Lyapunov function exists, then the hybrid system is stable for any switching. In the absence of the common Lyapunov function, stability properties of the hybrid system in general depend on the switching signal, and in this case the hybrid system is not stable for any switching rules but only for the so-called stabilizing switching rules [1]. In this case, more generally a single Lyapunov and a single Lyapunovlike functions have been introduced $[1,3,7]$. Some results for the linear stochastic hybrid systems are given in [8] and for the nonlinear deterministic hybrid systems are given in [9]. Recent results for the deterministic hybrid systems are collected and summarized in [10].

In the present paper, ideas of a feedback control, proposed by Florchinger for nonhybrid stochastic nonlinear control systems [11, 12], are used and combined with the concept of stabilizing switching rules for hybrid systems to derive the results for the $p$ th mean exponential stabilizability of stochastic nonlinear and bilinear hybrid systems consisting of unstable and stable structures. The authors propose also a design method for stabilizing switching rules, which is based on the knowledge of regions of decreasing the Lyapunov functions for subsystems. Similar methods were used for deterministic hybrid systems, for example, in [3, 6-8]. In this paper, we extend them to stochastic hybrid case.

\section{Mathematical Preliminaries}

Throughout this paper, we use the following notation. Let $|\cdot|$ be the Euclidean norm. By $\lambda(\mathbf{A})$ we denote the eigenvalue of the matrix $\mathbf{A}$, and $\lambda_{\min }(\mathbf{A})$ and $\lambda_{\max }(\mathbf{A})$ denote the smallest and the biggest eigenvalues of the matrix $\mathbf{A}$, respectively. We denote by $\mathbf{A}^{T}$ the transposition of matrix $\mathbf{A}$. We mark $\mathbb{T}=\left[t_{0}, \infty\right), t_{0} \geq 0$. Let $\Xi=\left(\Omega, \mathscr{F},\left\{\mathscr{F}_{t}\right\}_{t \geq 0}, \mathbb{P}\right)$ be a complete probability space with a filtration $\left\{\mathscr{F}_{t}\right\}_{t \geq 0}$ satisfying usual conditions. Let $\mathbf{w}(\mathbf{t})=\left[w_{1}(t), \ldots, w_{M}(t)\right]^{T}, t \geq 0$, be the $m$-dimensional standard Wiener process defined on the probability space $\Xi$. Let $\mathcal{S}=\{1, \ldots, N\}$ be the set of states, and let $\sigma(\cdot):\left[t_{0}, \infty\right) \rightarrow \mathcal{S}$ be the stochastic switching rule. 
We denote switching times as $\tau_{1}, \tau_{2}, \ldots$ and assume that there is a finite number of switches on every finite time interval. We assume that processes $w_{k}$ and $\sigma$ are both $\left\{\mathscr{F}_{t}\right\}_{t \geq 0}$ adapted. We say that a proper twice differentiable function $V: \mathscr{R}^{n} \rightarrow$ $\mathscr{R}_{+}=[0, \infty)$ is a Lyapunov function if $V(\mathbf{0})=0$ and $V(\mathbf{x})>$ 0 , for any $\mathbf{x} \neq 0$.

Let us consider the stochastic hybrid system described by the vector Itô differential equation

$$
\begin{gathered}
d \mathbf{x}(t)=\mathbf{f}(\mathbf{x}(t), t, \sigma) d t+\sum_{k=1}^{M} \mathbf{g}_{k}(\mathbf{x}(t), t, \sigma) d w_{k}(t), \\
\left(\sigma\left(t_{0}\right), \mathbf{x}\left(t_{0}\right)\right)=\left(\sigma_{0}, \mathbf{x}_{0}\right)
\end{gathered}
$$

where $\mathbf{x} \in \mathscr{R}^{n}$ is the state vector and $\left(\sigma_{0}, \mathbf{x}_{0}\right)$ is an initial condition, $t \in \mathbb{T}$. Functions $\mathbf{f}: \mathscr{R}^{n} \times \mathbb{T} \times \mathcal{S} \rightarrow \mathscr{R}^{n}$ and $\mathbf{g}_{k}: \mathscr{R}^{n} \times \mathbb{T} \times \mathcal{S} \rightarrow \mathscr{R}^{n}$ are locally Lipschitz such that $\forall l \in \mathcal{S}, t \in \mathbb{T} \mathbf{f}(\mathbf{0}, t, l)=\mathbf{g}_{k}(\mathbf{0}, t, l)=\mathbf{0}, k=1, \ldots, M$. The local Lipschitz conditions together with these enforced on the switching rule $\sigma$ ensure that there exists a unique solution to the hybrid system (1).

For any twice differentiable with respect to $\mathbf{x} \in \mathscr{R}^{n}$ and once differentiable with respect to $t \in \mathbb{T}$ function $\phi(\mathbf{x}, t, l)$ (i.e., $\left.\phi(\mathbf{x}, t, l) \in C^{2,1}\left(\mathscr{R}^{n} \times \mathbb{\mathbb { V }} \times \mathcal{S} ;[0, \infty)\right)\right)$, the $l$ th process has a generator $\mathscr{L}_{l}^{(1)}$ (the Itô operator for the $l$ th subsystem of the system (1)) given in every structure by

$$
\begin{aligned}
& \mathscr{L}_{l}^{(1)} \phi(\mathbf{x}, t, l)= \frac{\partial \phi(\mathbf{x}, t, l)}{\partial t}+\sum_{\mu=1}^{n} f^{\mu}(\mathbf{x}, t, l) \frac{\partial \phi(\mathbf{x}, t, l)}{\partial x_{\mu}} \\
&+\frac{1}{2} \sum_{r, s=1}^{n} \sum_{k=1}^{M} g_{k}^{r}(\mathbf{x}, t, l) g_{k}^{s}(\mathbf{x}, t, l) \frac{\partial^{2} \phi(\mathbf{x}, t, l)}{\partial x_{r} \partial x_{s}}, \\
& l \in \mathcal{S} .
\end{aligned}
$$

We use the following definitions.

Definition 1. The null solution $\mathbf{x} \equiv \mathbf{0}$ of the stochastic differential equation ( 1 ) is said to be $p$ th mean exponentially stable, $p>0$, if there exists a pair of positive scalars $\kappa, c$ such that

$$
\begin{gathered}
\forall\left(\mathbf{x}_{0}, t_{0}\right) \in \mathscr{R}^{n} \times[0, \infty), \\
\mathbb{E}\left[\left|\mathbf{x}\left(t, \mathbf{x}_{0}, t_{0}\right)\right|^{p}\right] \leq c \mathbb{E}\left[\left|\mathbf{x}_{0}\right|^{p}\right] \exp \left\{-\kappa\left(t-t_{0}\right)\right\}, \quad t \geq t_{0},
\end{gathered}
$$

where $\kappa$ is called the decay rate.

Definition 2. The hybrid system (1) is said to be stabilizable if there exist a switching signal $\sigma: \mathbb{T} \rightarrow \mathcal{S}$ and the associated linear feedback control law $\mathbf{u}: \mathscr{R}^{n} \times \mathbb{T} \times \mathcal{S} \rightarrow \mathscr{R}^{m}$ such that the hybrid system (1) is $p$ th mean exponentially stable for some $p>0$.

Definition 3. A Lyapunov function $V(\mathbf{x}) \in C^{2}\left(\mathscr{R}^{n} ; \mathscr{R}_{+}\right)$ satisfying

$$
\forall l \in \mathcal{S}, \forall \mathbf{x} \neq 0 \quad \mathscr{L}_{l}^{(1)} V(\mathbf{x})<0
$$

is called a common Lyapunov function for the hybrid system (1).

Note that it is a known fact that if there exists a common Lyapunov function for the hybrid system (1), then the null solution of (1) is asymptotically stable for any switching.

Definition 4. A Lyapunov function $V(\mathbf{x}) \in C^{2}\left(\mathscr{R}^{n} ; \mathscr{R}_{+}\right)$ satisfying

$$
\forall \mathbf{x} \neq 0 \quad \mathscr{L}_{\sigma(t)}^{(1)} V(\mathbf{x})<0
$$

for some switching rule $\sigma$ is called a single Lyapunov function for the hybrid system (1).

Lemma 5 ([13] (Itô formula)). If $\phi(\mathbf{x}, t, l) \in C^{2,1}\left(\mathscr{R}^{n} \times \mathbb{T} \times\right.$ $\mathcal{S} ;[0, \infty))$, then for any switching times $0 \leq \tau_{1} \leq \tau_{2}<\infty$,

$$
\begin{aligned}
\forall l \in \mathcal{S} \mathbb{E}\left[\phi\left(\mathbf{x}\left(\tau_{2}\right), \tau_{2}, l\right)\right]= & \mathbb{E}\left[\phi\left(\mathbf{x}\left(\tau_{1}\right), \tau_{1}, l\right)\right] \\
& +\mathbb{E}\left[\int_{\tau_{1}}^{\tau_{2}} \mathscr{L}_{l} \phi(\mathbf{x}(s), s, l) d s\right]
\end{aligned}
$$

if the integrations involved exist and are finite.

Following the methodology introduced in [6], for deterministic hybrid systems, we assume that the hybrid state space is partitioned into regions $\Omega_{l}, l \in \mathcal{S}$, and $\bigcup_{l \in \mathcal{S}} \Omega_{l}=\mathscr{R}^{n}$. We consider a special class of switching rule $\mathcal{S} \mathscr{W}$ given by

$$
\mathcal{S} \mathscr{W}=\left\{\sigma:(\sigma(t)=l) \Longrightarrow\left(\mathbf{x}(t) \in \Omega_{l}\right), l \in \mathcal{S}\right\}
$$

Note that a switching rule $\sigma \in \mathcal{S} \mathscr{W}$ given by (7) is a stochastic switching rule because of its dependence on the stochastic process $\mathbf{x}(t)$. Our aim is to find a special partition $\Omega_{l}$ defined by (7) such that every switching rule $\sigma \in \mathcal{S} \mathscr{W}$ is a stabilizing switching rule for a considered class of stochastic hybrid systems.

\section{Stability of Nonlinear Stochastic Hybrid Systems}

First, we study the problem of the stability of the nonlinear stochastic hybrid system (1). Two cases, single Lyapunov and single Lyapunov-like functions, are considered.

3.1. Single Lyapunov Functions. We formulate a theorem which establishes sufficient conditions for the $p$ th mean exponential stability of the nonlinear hybrid system (1).

Theorem 6. If the following conditions hold:

(1) there exist a Lyapunov function $V: \mathscr{R}^{n} \rightarrow \mathscr{R}_{+}$and positive constants $C_{\min }, C_{\max }$ such that

$$
C_{\min }|\mathbf{x}|^{p} \leq V(\mathbf{x}) \leq C_{\max }|\mathbf{x}|^{p}, \quad p>0
$$


(2) there exists a Lebesgue-measurable function $\kappa: \mathbb{T} \rightarrow$ $(0, \infty)$ such that

$$
\bigcup_{l \in \mathcal{S}} \Omega_{l}=\mathscr{R}^{n} \text {, where }
$$

$\Omega_{l}=\left\{\mathbf{x} \in \mathscr{R}^{n}: \forall t \in \mathbb{T} \mathscr{L}_{l}^{(1)} V(\mathbf{x}) \leq-\kappa(t) V(\mathbf{x})\right\} \quad$ for $l \in \mathcal{S}$.

Then the null solution $\mathbf{x} \equiv \mathbf{0}$ of the stochastic hybrid system (1) is pth mean exponentially stable under the stabilizing switching rule $\sigma_{\text {st }} \in \mathcal{S} \mathscr{W}$.

Proof. From assumptions for the Lyapunov function $V$ : $\mathscr{R}^{n} \rightarrow \mathscr{R}_{+}$, we obtain

$$
\begin{gathered}
C_{\min }|\mathbf{x}|^{p} \leq V(\mathbf{x}) \leq C_{\max }|\mathbf{x}|^{p}, \quad p>0, \\
\mathbb{E}\left[\mathscr{L}_{\sigma_{\mathrm{st}}}^{(1)} V(\mathbf{x})\right] \leq-\kappa(t) \mathbb{E}[V(\mathbf{x})] .
\end{gathered}
$$

From (11) and the Itô formula 6 , we obtain

$$
\begin{aligned}
\mathbb{E}[V(\mathbf{x})] & =\mathbb{E}\left[V\left(\mathbf{x}_{0}\right)\right]+\mathbb{E}\left[\int_{t_{0}}^{t} \mathscr{L}_{\sigma_{\mathrm{st}}}^{(1)} V(\mathbf{x}(s)) d s\right] \\
& \leq \mathbb{E}\left[V\left(\mathbf{x}_{0}\right)\right]-\int_{t_{0}}^{t} \kappa(t) \mathbb{E}[V(\mathbf{x}(s))] d s
\end{aligned}
$$

and by Gronwall's inequality

$$
E[V(\mathbf{x})] \leq E\left[V\left(\mathbf{x}_{0}\right)\right] \exp \left(-\int_{t_{0}}^{t} \kappa(t) d s\right) .
$$

Now from (10) and (13), we obtain the following inequality:

$$
E\left[|\mathbf{x}(t)|^{p}\right] \leq \frac{C_{\max }}{C_{\min }} E\left[\left|\mathbf{x}_{0}\right|^{p}\right] \exp \left(-\int_{t_{0}}^{t} \kappa(t) d s\right), \quad p>0 .
$$

Hence, the thesis follows.

Notice that function $V$ is a single Lyapunov function for the hybrid system (1).

3.2. Single Lyapunov-Like Functions. In the case when condition (2) of Theorem 6 is not satisfied, then one can look for a single Lyapunov-like function. We assume in this case that the hybrid state space is partitioned into regions $\Omega_{l}$ and $l \in \mathcal{S}$, which can be separated into two disjoint subregions: a stable subregion $\Omega_{l}^{\mathrm{s}}$ and an unstable region $\Omega_{l}^{\text {us }}, l \in \mathcal{S}$ (the upper scripts "s" and "us" denote stable and unstable regions, resp.) that is,

$$
\Omega_{l}=\Omega_{l}^{\mathrm{s}} \cup \Omega_{l}^{\mathrm{us}}, \quad \Omega_{l}^{\mathrm{s}} \cap \Omega_{l}^{\mathrm{us}}=\emptyset .
$$

Let us denote by $\mathbb{T}_{s}$ the sum of time intervals of the residence of the system (1) in the regions $\Omega_{l}^{s}, l \in \mathcal{S}$, and by $\mathbb{T}_{\text {us }}$ the sum of time intervals of the residence of the system (1) in the regions $\Omega_{l}^{\text {us }}, l \in \mathcal{S}$.

In this case we cannot construct a single Lyapunov function, but we can look for a single Lyapunov-like function defined as follows.
Definition 7. A Lyapunov function $V(\mathbf{x}) \in C^{2}\left(\mathscr{R}^{n} ; \mathscr{R}_{+}\right)$is called a single Lyapunov-like function if there exist positive constants $\kappa_{\mathrm{s}}$ and $\kappa_{\mathrm{us}}$ such that

$$
\mathscr{L}_{\sigma(t)}^{(1)} V(\mathbf{x}) \leq \begin{cases}-\kappa_{\mathrm{s}} V(\mathbf{x}) & \text { for } \mathbf{x} \in \Omega_{\sigma(t)}^{\mathrm{s}}, \\ \kappa_{\mathrm{us}} V(\mathbf{x}) & \text { for } \mathbf{x} \in \Omega_{\sigma(t)}^{\mathrm{us}},\end{cases}
$$

for some switching rule $\sigma$.

Using this definition, the following theorem can be formulated.

Theorem 8. Let one assume that the following conditions hold:

(1) there exist a Lyapunov function $V(\mathbf{x}) \in C^{2}\left(\mathscr{R}^{n} ; \mathscr{R}_{+}\right)$ and positive constants $C_{\min }, C_{\max }$ such that

$$
C_{\min }|\mathbf{x}|^{p} \leq V(\mathbf{x}) \leq C_{\max }|\mathbf{x}|^{p}, \quad p>0
$$

(2) there exist Lebesgue-measurable functions $\kappa_{\mathrm{s}}: \mathbb{T} \rightarrow$ $(0, \infty)$ and $\kappa_{\mathrm{us}}: \mathbb{T} \rightarrow(0, \infty)$ such that partition $(15)$ satisfies the conditions

$$
\bigcup_{l \in \mathcal{S}} \Omega_{l}=\mathscr{R}^{n} \text {, where }
$$

$$
\begin{aligned}
& \Omega_{l}^{\mathrm{s}}=\left\{\mathbf{x} \in \mathscr{R}^{n}: \forall t \in \mathbb{T} \mathscr{L}_{l}^{(1)} V(\mathbf{x}) \leq-\kappa_{\mathrm{s}}(t) V(\mathbf{x})\right\} \quad \text { for } l \in \mathcal{S}, \\
& \Omega_{l}^{\mathrm{us}}=\left\{\mathbf{x} \in \mathscr{R}^{n}: \forall t \in \mathbb{T} \quad \mathscr{L}_{l}^{(1)} V(\mathbf{x}) \leq \kappa_{\mathrm{us}}(t) V(\mathbf{x})\right\} \quad \text { for } l \in \mathcal{S} \text {, }
\end{aligned}
$$

(3) there exists a Lebesguemeasurable function $\kappa: \mathbb{T} \rightarrow$ $(0, \infty)$ such that $\mathscr{T} \neq \emptyset$, where

$$
\mathscr{T}=\left\{\sigma \in \mathcal{S} \mathscr{W}: \int_{T_{\mathrm{us}}}\left[\kappa_{\mathrm{us}}(s)+\kappa(s)\right] d s \leq \int_{T_{\mathrm{s}}}\left[\kappa_{\mathrm{s}}(s)-\kappa(s)\right] d s\right\} .
$$

Then the null solution $\mathbf{x} \equiv \mathbf{0}$ of (1) is pth mean exponentially stable under the stabilizing switching rule $\bar{\sigma}_{\text {st }} \in \mathscr{T}$.

Proof. From assumptions for $\sigma_{\text {st }} \in \mathcal{S} \mathscr{W}$, it follows that

$$
\mathbb{E}[V(\mathbf{x})] \leq \mathbb{E}\left[V\left(\mathbf{x}_{0}\right)\right] \exp \left(\int_{T_{\text {us }}} \kappa_{\text {us }}(s) d s-\int_{T_{\mathrm{s}}} \kappa_{\mathrm{s}}(s) d s\right) .
$$

Let us consider the switching strategy $\bar{\sigma}_{\text {st }} \in \mathscr{T}$ described by (19). Then we obtain

$$
\mathbb{E}[V(\mathbf{x})] \leq \mathbb{E}\left[V\left(\mathbf{x}_{0}\right)\right] \exp \left(-\int_{t_{0}}^{t} \kappa(s) d s\right)
$$

Further proof is similar to the proof of Theorem 6. Hence, the thesis follows.

Notice that function $V$ is a single Lyapunov-like function for the hybrid system (1). 
Example 9. Let us consider a special case of the system (1) with the two subsystems $(\mathcal{S}=\{1,2\})$ given as follows:

$$
\begin{gathered}
d \mathbf{x}(t)=[\mathbf{A}(\sigma) \mathbf{x}(t)+\mathbf{f}(\mathbf{x}, \sigma)] d t+\mathbf{G}(\sigma) d w(t), \\
\left(\sigma\left(t_{0}\right), \mathbf{x}\left(t_{0}\right)\right)=\left(\sigma_{0}, \mathbf{x}_{0}\right) \in \mathcal{S} \times \mathscr{R}^{n},
\end{gathered}
$$

where

$$
\begin{aligned}
& \mathbf{A}(1)=\left[\begin{array}{cc}
2.18 & 2 \\
-4 & -2.82
\end{array}\right], \quad \mathbf{A}(2)=\left[\begin{array}{cc}
-1.905 & 4 \\
-2 & 1.095
\end{array}\right], \\
& \mathbf{f}(\mathbf{x}, 1)=\left[\begin{array}{c}
0 \\
f_{2}\left(x_{2}, 1\right)
\end{array}\right], \quad \mathbf{f}(\mathbf{x}, 2)=\left[\begin{array}{c}
0 \\
f_{2}\left(x_{2}, 2\right)
\end{array}\right], \\
& f_{2}\left(x_{2}, 1\right)= \begin{cases}0.8 x_{2}-0.2 & \text { for } x_{2}<-1, \\
x_{2} & \text { for } x_{2} \in[-1,1] \\
0.8 x_{2}+0.2 & \text { for } x_{2}>1\end{cases} \\
& f_{2}\left(x_{2}, 2\right)= \begin{cases}0.9 x_{2}-0.1 & \text { for } x_{2}<-1, \\
x_{2} & \text { for } x_{2} \in[-1,1] \\
0.9 x_{2}+0.1 & \text { for } x_{2}>1,\end{cases} \\
& \mathbf{G}(1)=\left[\begin{array}{cc}
0.2 & 0 \\
0 & 0.2
\end{array}\right], \quad \mathbf{G}(2)=\left[\begin{array}{cc}
0.1 & 0 \\
0 & 0.1
\end{array}\right] \text {. }
\end{aligned}
$$

Let us consider the Lyapunov function $V(\mathbf{x})=\mathbf{x}^{T} \mathbf{x}$. Since $0 \leq f_{2}\left(x_{2}, l\right) x_{2} \leq x_{2}^{2}, l=1,2$, then

$$
\begin{gathered}
\mathscr{L}_{1}^{(22)} V(\mathbf{x})=2.2 x_{1}^{2}-2 x_{1} x_{2}-2.8 x_{2}^{2}+f(\mathbf{x}, 1)^{T} \mathbf{x} \\
\leq-0.8\left(x_{1}^{2}+x_{2}^{2}\right) \text { for } \\
\Omega_{1}^{\mathrm{s}}=\left\{\mathbf{x} \in \mathscr{R}^{2}:\left(x_{2}+3 x_{1}\right)\left(x_{1}-x_{2}\right) \leq 0\right\}, \\
\mathscr{L}_{2}^{(22)} V(\mathbf{x})=-1.9 x_{1}^{2}+2 x_{1} x_{2}+1.1 x_{2}^{2}+f(\mathbf{x}, 2)^{T} \mathbf{x} \\
\leq-0.8\left(x_{1}^{2}+x_{2}^{2}\right) \text { for } \\
\Omega_{2}^{\mathrm{s}}=\left\{\mathbf{x} \in \mathscr{R}^{2}:-1.1 x_{1}^{2}+2 x_{1} x_{2}+2.9 x_{2}^{2} \leq 0\right\}, \\
\mathscr{L}_{2}^{(22)} V(\mathbf{x})=-1.9 x_{1}^{2}+2 x_{1} x_{2}+1.1 x_{2}^{2}+f(\mathbf{x}, 2)^{T} \mathbf{x} \\
\leq 1.1\left(x_{1}^{2}+x_{2}^{2}\right) \text { for } \\
\Omega_{2}^{\mathrm{us}}=\left\{\mathbf{x} \in \mathscr{R}^{2}:\left(x_{2}+3 x_{1}\right)\left(x_{2}-x_{1}\right) \leq 0\right\} \backslash\left(\Omega_{1}^{\mathrm{s}} \cup \Omega_{2}^{\mathrm{s}}\right) .
\end{gathered}
$$

Note that $\bigcup_{l \in \mathcal{S}} \Omega_{l}=\mathscr{R}^{2}$ and functions $\kappa_{\mathrm{s}}$ and $\kappa_{\mathrm{us}}$ are constant and are given as follows $\kappa_{\mathrm{s}}=0.8, \kappa_{\mathrm{us}}=1.1$.

Condition

$$
\frac{\mathbb{T}_{\mathrm{us}}}{\mathbb{T}_{\mathrm{s}}} \leq \frac{\kappa_{\mathrm{s}}-\kappa}{\kappa_{\mathrm{us}}+\kappa} \approx 0.61
$$

is satisfied for $\kappa=0.08$. Exemplary simulations are shown in Figures 1, 2, and 3.

From Theorem 8, it follows that $V(\mathbf{x})=\mathbf{x}^{T} \mathbf{x}$ is the single Lyapunov-like function for the system (22), and it is exponentially stable in mean square with a decay rate $\kappa=0.08$ for the switching strategy $\bar{\sigma}_{\text {st }} \in \mathscr{T}$ given by

$$
\bar{\sigma}_{\text {st }}(\mathbf{x}, t)= \begin{cases}1 & \text { if } \mathbf{x}(t) \in \Omega_{1} \\ 2 & \text { if } \mathbf{x}(t) \in \Omega_{2}\end{cases}
$$

\section{Stabilizability of Stochastic Hybrid Systems}

In this section, we discuss the stabilizability problem of the stochastic nonlinear and bilinear hybrid systems. We formulate sufficient conditions for the $p$ th mean exponential stabilizability, and we find a control of feedback form for the considered class of systems.

4.1. Stabilizability of Nonlinear Stochastic Hybrid Systems. Let us consider the stochastic control hybrid system described by the vector Itô differential equations

$$
\begin{aligned}
d \mathbf{x}(t)=\mathbf{f}_{0}(\mathbf{x}(t), t, \sigma) d t+\sum_{i=1}^{p} u_{i}(\mathbf{x}, t, \sigma) \mathbf{f}_{i}(\mathbf{x}(t), t, \sigma) \\
+\sum_{k=1}^{M} \mathbf{g}_{k}(\mathbf{x}(t), t, \sigma) d w_{k}(t) \\
\left(\sigma\left(t_{0}\right), \mathbf{x}\left(t_{0}\right)\right)=\left(\sigma_{0}, \mathbf{x}_{0}\right),
\end{aligned}
$$

where $\mathbf{x} \in \mathscr{R}^{n}$ is the state vector, $\mathbf{u}=\left[u_{1}, \ldots, u_{m}\right]^{T}$ is a measurable $\mathscr{R}^{m}$ - a real-valued control vector law, $\left(\sigma_{0}, \mathbf{x}_{0}\right) \in$ $\mathcal{S} \times \mathscr{R}^{n}$ is an initial condition, and $t \in \mathbb{T}$. Functions $\mathbf{f}_{i}$ : $\mathscr{R}^{n} \times \mathbb{\mathbb { N }} \times \mathcal{S} \rightarrow \mathscr{R}^{n}$ and $\mathbf{g}_{k}: \mathscr{R}^{n} \times \mathbb{\mathbb { N }} \times \mathcal{S} \rightarrow \mathscr{R}^{n}$ are the locally Lipschitz, $\mathbf{f}_{i}(\mathbf{0}, t, l)=\mathbf{g}_{k}(\mathbf{0}, t, l)=\mathbf{0}, \forall l \in \mathcal{S}, t \in \mathbb{T}$, $i=0, \ldots, m, k=1, \ldots, M$.

The local Lipschitz condition together with these enforced on the switching rule $\sigma$ ensures that there is a unique solution to the hybrid system (27).

The aim of this part of the paper is to establish sufficient conditions under which one can design a state feedback control law so that the null solution $\mathbf{x} \equiv \mathbf{0}$ of the stochastic hybrid control system (27) is pth mean exponentially stable. We extend the results of Florchinger for the stochastic nonhybrid systems $[11,12]$ to the hybrid systems. Some results for asymptotic stability and stabilizability for the hybrid system (27) with Markovian or any switchings under a feedback control have been proposed in [14].

We introduce the following notation of operators $L_{l}$ and $L_{l}^{i}, l \in \mathcal{S}, i=1, \ldots, m$, for $\phi(\mathbf{x}, t, l) \in C^{2,1}\left(\mathscr{R}^{n} \times \mathbb{T} \times \mathcal{S} ; \mathscr{R}_{+}\right)$:

$$
\begin{aligned}
L_{l} \phi(\mathbf{x}, t, l)= & \frac{\partial \phi(\mathbf{x}, t, l)}{\partial t}+\sum_{\mu=1}^{n} f_{0}^{\mu}(\mathbf{x}, t, l) \frac{\partial \phi(\mathbf{x}, t, l)}{\partial x_{\mu}} \\
& +\frac{1}{2} \sum_{r, \mathrm{~s}=1}^{n} \sum_{k=1}^{M} g_{k}^{r}(\mathbf{x}, t, l) g_{k}^{\mathrm{s}}(\mathbf{x}, t, l) \frac{\partial^{2} \phi(\mathbf{x}, t, l)}{\partial x_{r} \partial x_{\mathrm{s}}},
\end{aligned}
$$

$L_{l}^{i} \phi(\mathbf{x}, t, l)=\sum_{\mu=1}^{n} f_{i}^{\mu}(\mathbf{x}, t, l) \frac{\partial \phi(\mathbf{x}, t, l)}{\partial x_{\mu}}, \quad i=1, \ldots, m, l \in \mathcal{S}$. 


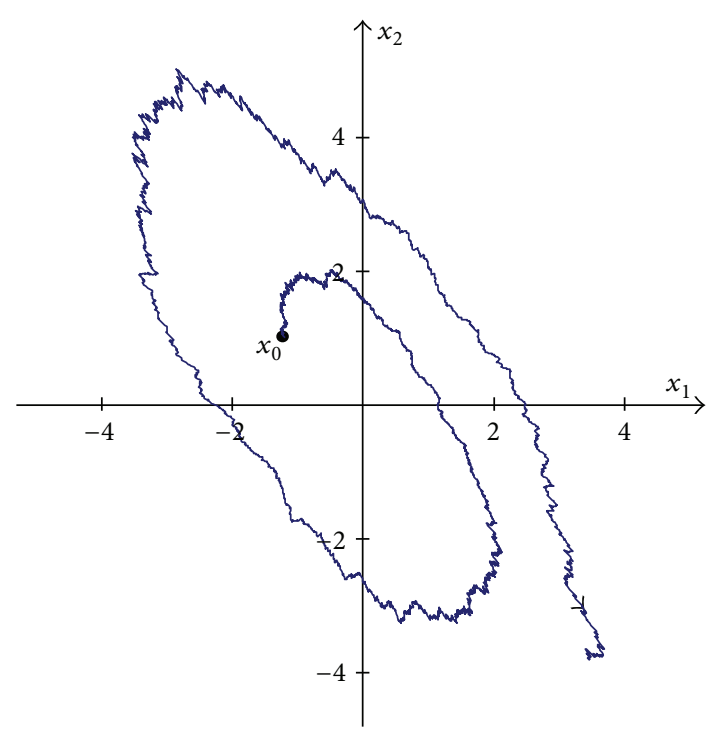

(a) Unstable path of the system (22) for $l=1$

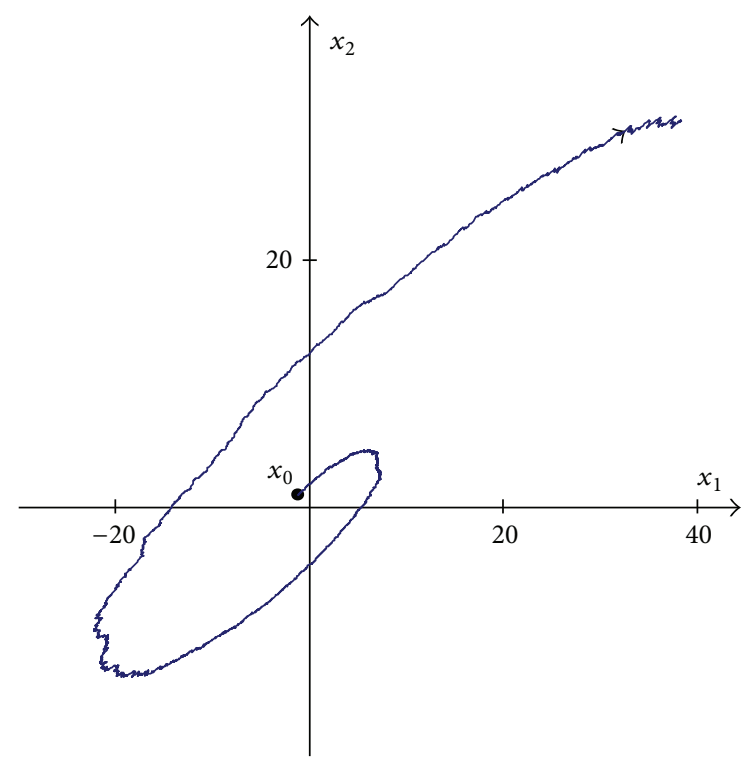

(b) Unstable path of the system (22) for $l=2$

FIGURE 1: Trajectories of the subsystems of (22).

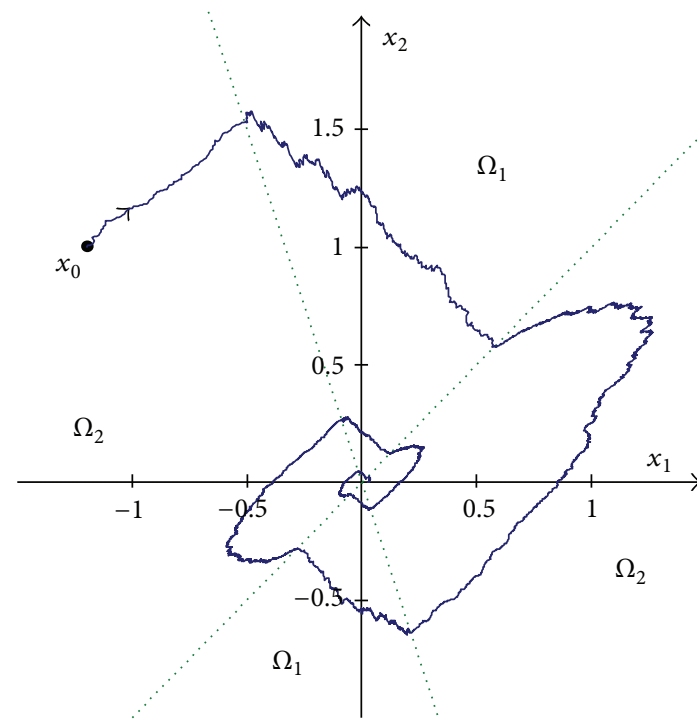

(a) Stable path of the hybrid system (22) with stabilizing switching $\bar{\sigma}_{\text {st }}$ given by (26)

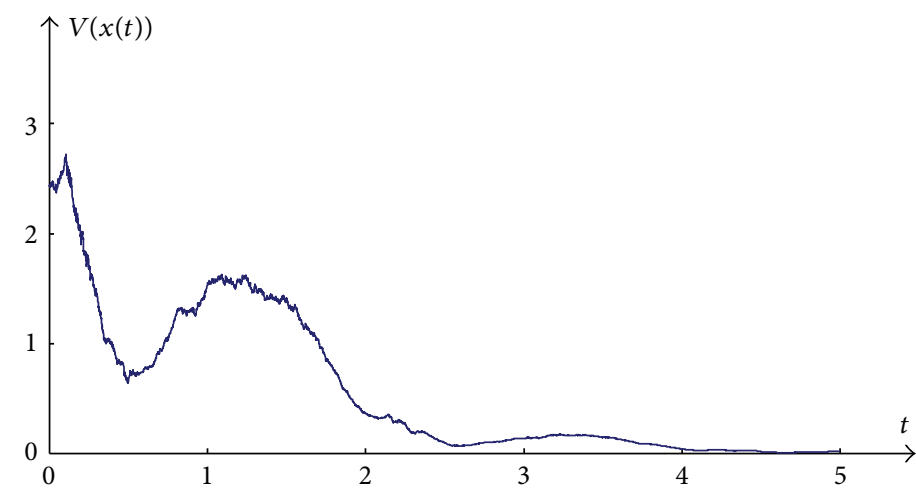

(b) Lyapunov function $V=\mathbf{x}^{T} \mathbf{x}$ for the hybrid system (22)

FIgURE 2: Trajectories of the hybrid system (22).

Then, the following stabilization result for the control hybrid system (27) holds.

Theorem 10. Suppose that the following conditions hold:

(1) there exist a Lyapunov function $V(\mathbf{x}) \in C^{2}\left(\mathscr{R}^{n} ; \mathscr{R}_{+}\right)$ and positive constants $C_{\min }, C_{\max }$ such that

$$
C_{\min }|\mathbf{x}|^{p} \leq V(\mathbf{x}) \leq C_{\max }|\mathbf{x}|^{p}, \quad p>0,
$$

(2) there exists a Lebesgue-measurable function $\kappa: \mathbb{T} \rightarrow$ $(0, \infty)$ such that partition determined by (7) satisfies conditions

$$
\bigcup_{l \in \mathcal{S}} \Omega_{l}=\mathscr{R}^{n} \text {, where }
$$

$\Omega_{l}=\left\{\mathbf{x} \in \mathscr{R}^{n}: \forall t \in \mathbb{T} \quad L_{l} V(\mathbf{x}) \leq-\kappa(t) V(\mathbf{x})\right\} \quad$ for $l \in \mathcal{S}$.

Then the control law $\mathbf{u}: \mathscr{R}^{n} \times \mathbb{T} \times \mathcal{S} \rightarrow \mathscr{R}^{m}$ given as follows:

$$
u_{i}(\mathbf{x}, t, l)=-L_{l}^{i} V(\mathbf{x}), \quad i=1,2, \ldots, m, l \in \mathcal{S}
$$




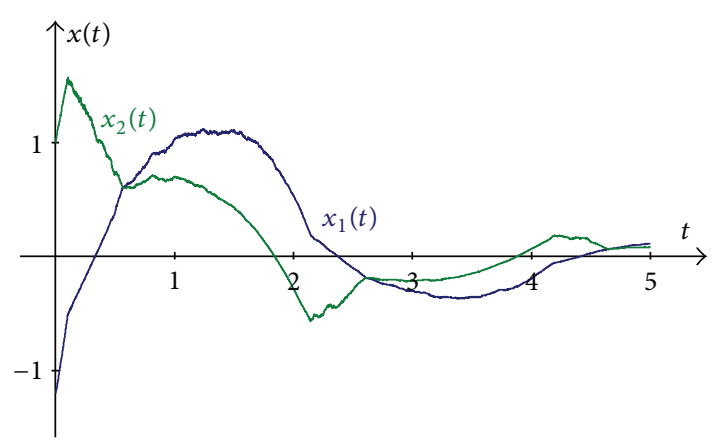

(a)

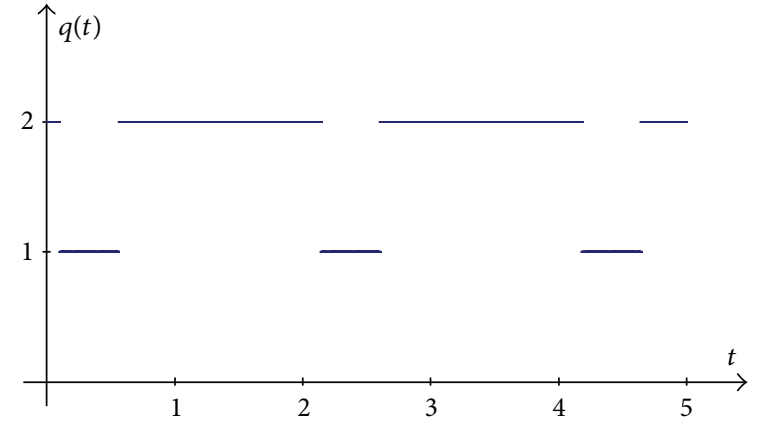

(b)

FIGURE 3: Stable path of the hybrid system (22) with stabilizing switching $\bar{\sigma}_{\text {st }}$ given by (26).

together with the stabilizing switching rule $\sigma_{\text {st }} \in \mathcal{S} \mathscr{W}$ renders the null solution of the stochastic hybrid system (27) the pth mean exponentially stable.

Proof. Applying the infinitesimal operator $\mathscr{L}$ defined by (2) to the hybrid system (27), we find that

$$
\begin{aligned}
\forall l \in \mathcal{S} \quad \mathscr{L}_{l}^{(27)} V(\mathbf{x}) & =L_{l} V(\mathbf{x})-\sum_{i=1}^{m}\left(L_{l}^{i} V(\mathbf{x})\right)^{2} \\
& \leq-\kappa(t) V(\mathbf{x}), \quad \text { for } \mathbf{x} \in \Omega_{l} .
\end{aligned}
$$

Now the thesis follows from Theorem 6.

We can formulate a more general theorem in a case when a Lyapunov-like function exists as follows.

Theorem 11. Suppose that the following conditions hold:

(1) there exist a Lyapunov function $V(\mathbf{x}) \in C^{2}\left(\mathscr{R}^{n} ; \mathscr{R}_{+}\right)$ and positive constants $C_{\min }, C_{\max }$ such that

$$
C_{\min }|\mathbf{x}|^{p} \leq V(\mathbf{x}) \leq C_{\max }|\mathbf{x}|^{p}, \quad p>0
$$

(2) there exist Lebesgue-measurable functions $\kappa_{\mathrm{s}}: \mathbb{T} \rightarrow$ $(0, \infty)$ and $\kappa_{\mathrm{us}}: \mathbb{T} \rightarrow(0, \infty)$ such that partition $(15)$ satisfies conditions

$$
\bigcup_{l \in \mathcal{S}} \Omega_{l}=\mathscr{R}^{n} \text {, where }
$$

$\Omega_{l}^{\mathrm{s}}=\left\{\mathbf{x} \in \mathscr{R}^{n}: \forall t \in \mathbb{T} \quad L_{l} V(\mathbf{x}) \leq-\kappa_{\mathrm{s}}(t) V(\mathbf{x})\right\} \quad$ for $l \in \mathcal{S}$, $\Omega_{l}^{\mathrm{us}}=\left\{\mathbf{x} \in \mathscr{R}^{n}: \forall t \in \mathbb{T} \quad L_{l} V(\mathbf{x}) \leq \kappa_{\mathrm{us}}(t) V(\mathbf{x})\right\} \quad$ for $l \in \mathcal{S}$,

(3) there exists a Lebesgue-measurable function $\kappa: \mathbb{T} \rightarrow$ $\mathscr{R}_{+}$such that $\mathscr{T} \neq \emptyset$, where

$$
\mathscr{T}=\left\{\sigma \in \mathcal{S} \mathscr{W}: \int_{T_{\mathrm{us}}}\left[\kappa_{\mathrm{us}}(s)+\kappa(s)\right] d s \leq \int_{T_{\mathrm{s}}}\left[\kappa_{\mathrm{s}}(s)-\kappa(s)\right] d s\right\} .
$$

Then the control law $\mathbf{u}: \mathscr{R}^{n} \times \mathbb{\mathbb { V }} \times \mathcal{S} \rightarrow \mathscr{R}^{m}$ given as follows

$$
u_{i}(\mathbf{x}, t, l)=-L_{l}^{i} V(\mathbf{x}), \quad i=1,2, \ldots, m
$$

together with the stabilizing switching rule $\bar{\sigma}_{\text {st }} \in \mathscr{T}$ renders the null solution of the stochastic hybrid system (27) the pth mean exponentially stable.

Proof. The thesis follows from Theorem 8.

4.2. Stabilizability of the Bilinear Hybrid Systems. Let us consider a special class of the system (27) given by a bilinear stochastic hybrid system as follows:

$$
\begin{gathered}
d \mathbf{x}(t)=\left[\mathbf{A}(\sigma(t)) \mathbf{x}(t)+\sum_{i=1}^{M} u_{i}(\mathbf{x}(t), \sigma(t)) \mathbf{C}_{i}(\sigma(t)) \mathbf{x}(t)\right] d t \\
+\sum_{k=1}^{M} \mathbf{B}_{k}(\sigma(t)) \mathbf{x}(t) d w_{k}(t), \\
\left(\sigma\left(t_{0}\right), \mathbf{x}\left(t_{0}\right)\right)=\left(\sigma_{0}, \mathbf{x}_{0}\right),
\end{gathered}
$$

where $\mathbf{x} \in \mathscr{R}^{n}$ is the state vector, $\mathbf{u}=\left[u_{1}, \ldots, u_{m}\right]^{T} \in \mathscr{R}^{m}, u_{i}$ : $\mathscr{R}^{n} \times \mathcal{S} \rightarrow \mathscr{R}$, is the control vector, $t \in \mathbb{T},\left(\sigma_{0}, \mathbf{x}_{0}\right) \in \mathcal{S} \times \mathscr{R}^{n}$ is an initial condition, and $\mathbf{A}(l), \mathbf{C}_{i}(l), \mathbf{B}_{k}(l), i=1, \ldots, m, k=$ $1, \ldots, M$ are for every $l \in \mathcal{S}$ constant matrices of dimension $n \times n$.

For this particular case, we can combine the above results with the theorem given by Mao [15] for the stochastic linear systems and formulate the theorems which can be obtained directly from Theorems 10 and 11. Sufficient conditions for the $p$ th mean exponential stabilizability for the linear hybrid systems are formulated in [16].

Operators (28) reduce to the following ones:

$$
\begin{gathered}
L_{l} \phi(\mathbf{x}, l)=\mathbf{x}^{T} \mathbf{A}^{T}(l) \nabla \phi(\mathbf{x}, l) \\
+\frac{1}{2} \operatorname{tr}\left(\mathbf{B}_{k}(l) \mathbf{x} \mathbf{x}^{T} \mathbf{B}_{k}(l)^{T} \nabla^{2} \phi(\mathbf{x}, l)\right), \\
l \in \mathcal{S}, \\
L_{l}^{i}(\mathbf{x}, l)=\mathbf{x}^{T} \mathbf{C}_{i}^{T}(l) \nabla \phi(\mathbf{x}, l), \quad i=1, \ldots, m, l \in \mathcal{S},
\end{gathered}
$$

where $\nabla \phi(\cdot, q)$ and $\nabla^{2} \phi(\cdot, q)$ denote gradient and Hessian of the function $\phi(\cdot, q)$, respectively. 
Theorem 12. Suppose that there exist symmetric positive definite matrix $\mathbf{H}$, constant $\alpha_{1}$, and positive constants $\alpha_{2}, \alpha_{3}$ such that the following conditions are satisfied:

$$
\begin{gathered}
\bigcup_{l \in \mathcal{S}} \Omega_{l}=\mathscr{R}^{n} \text {, where } \\
\Omega_{l}=\left\{\mathbf{x} \in \mathscr{R}^{n}: \mathbf{x}^{T} \mathbf{A}^{T}(l) \mathbf{H} \mathbf{x}+\frac{1}{2} \sum_{k=1}^{M} \mathbf{x}^{T} \mathbf{B}_{k}^{T}(l) \mathbf{H B}_{k}(l) \mathbf{x}\right. \\
\leq \alpha_{1} \mathbf{x}^{T} \mathbf{H} \mathbf{x} \wedge \alpha_{2} \mathbf{x}^{T} \mathbf{H} \mathbf{x} \\
\left.\leq\left|\mathbf{x}^{T} \mathbf{H} \sum_{k=1}^{M} \mathbf{B}_{k}(l) \mathbf{x}\right| \leq \alpha_{3} \mathbf{x}^{T} \mathbf{H} \mathbf{x}\right\} \quad l \in \mathcal{S} .
\end{gathered}
$$

Then control $\mathbf{u}: \mathscr{R}^{n} \times \mathcal{S} \rightarrow \mathscr{R}^{m}$ of a form

$u_{i}(\mathbf{x}, l)=-p\left(\mathbf{x}^{T} \mathbf{H} \mathbf{x}\right)^{(p / 2)-1} \mathbf{x}^{T} \mathbf{C}_{i}^{T}(l) \mathbf{H} \mathbf{x}, \quad i=1,2, \ldots, m, l \in \mathcal{S}$

together with the stabilizing switching rule $\sigma_{\text {st }} \in \delta \mathscr{W}$ makes the hybrid system (37) the pth mean exponentially stable for

(a) $2 \leq p<2+2\left|\alpha_{1}\right| /\left(\alpha_{3}\right)^{2}$ if $\alpha_{1}<0$,

(b) $0<p<2-2 \alpha_{1} /\left(\alpha_{2}\right)^{2}$ if $0 \leq \alpha_{1}<\alpha_{2}^{2}$.

Proof. The thesis of the theorem follows from Theorem 10. Let us choose a Lyapunov function of a form

$$
V(\mathbf{x})=\left(\mathbf{x}^{T} \mathbf{H} \mathbf{x}\right)^{(p / 2)}, \quad p>0 .
$$

Notice that $V$ satisfies assumption (1) of Theorem 10 for $C_{\min }=\lambda_{\min }^{(p / 2)}(\mathbf{H})$ and $C_{\max }=\lambda_{\max }^{(p / 2)}(\mathbf{H})$. Then using assumptions (39), we obtain

$$
\forall l \in \mathcal{S} \quad L_{l} V(\mathbf{x}) \leq-\kappa V(\mathbf{x}) \quad \kappa>0 \quad \text { for } \mathbf{x} \in \Omega_{l},
$$

where

$$
\kappa=\left\{\begin{array}{rr}
p\left(\left|\alpha_{1}\right|-\left(\frac{p}{2}-1\right) \alpha_{3}^{2}\right) V(\mathbf{x}), & \text { for } 2 \leq p<2+\frac{2\left|\alpha_{1}\right|}{\left(\alpha_{3}\right)^{2}}, \\
\alpha_{1}<0, \\
p\left(-\alpha_{1}-\left(\frac{p}{2}-1\right) \alpha_{2}^{2}\right) V(\mathbf{x}), & \text { for } 0<p<2-\frac{2 \alpha_{1}}{\left(\alpha_{2}\right)^{2}}, \\
0 \leq \alpha_{1}<\left(\alpha_{2}\right)^{2} .
\end{array}\right.
$$

From Theorem 10, it follows that the control chosen as follows:

$$
\begin{array}{r}
u_{i}(\mathbf{x}, l)=-L_{l}^{i} V(\mathbf{x})=-p\left(\mathbf{x}^{T} \mathbf{H} \mathbf{x}\right)^{(p / 2)-1} \mathbf{x}^{T} \mathbf{C}_{i}^{T}(l) \mathbf{H} \mathbf{x}, \\
i=1,2, \ldots, m, l \in \mathcal{S}
\end{array}
$$

together with the stabilizing switching rule $\sigma_{\text {st }} \in \mathcal{S} \mathscr{W}$ makes the system (37) the pth mean exponentially stable for
(a) $2 \leq p<2+2\left|\alpha_{1}\right| /\left(\alpha_{3}\right)^{2}$ if $\alpha_{1}<0$,
(b) $0<p<2-2 \alpha_{1} /\left(\alpha_{2}\right)^{2}$ if $0 \leq \alpha_{1}<\alpha_{2}^{2}$.

Hence, the thesis follows.

Notice that function $V(\mathbf{x})=\left(\mathbf{x}^{T} \mathbf{H x}\right)^{(p / 2)}$ is a single Lyapunov function for the hybrid system (37). See [16] for the details of the proof.

Remark 13. In a particular case of $p=2$, we obtain the following criterion.

Criterion 14. Suppose that there exists a symmetric positive definite matrix $\mathbf{H}$ such that

$$
\begin{gathered}
\bigcup_{l \in \mathcal{S}} \Omega_{l}=\mathscr{R}^{n}, \text { where } \\
\Omega_{l}=\left\{\mathbf{x} \in \mathscr{R}^{n}: \mathbf{x}^{T} \mathbf{A}^{T}(l) \mathbf{H} \mathbf{x}+\frac{1}{2} \sum_{k=1}^{M} \mathbf{x}^{T} \mathbf{B}_{k}^{T}(l) \mathbf{H B}_{k}(l) \mathbf{x}<0\right\}, \\
l \in \mathcal{S} .
\end{gathered}
$$

Then the control u : $\mathscr{R}^{n} \times \mathcal{S} \rightarrow \mathscr{R}^{m}$ of a form

$$
u_{i}(\mathbf{x}, l)=-2 \mathbf{x}^{T} \mathbf{C}_{i}^{T}(l) \mathbf{H} \mathbf{x}, \quad i=1,2, \ldots, m, l \in \mathcal{S}
$$

together with the stabilizing switching rule $\sigma_{\text {st }} \in \delta \mathscr{W}$ exponentially in mean-square stabilizes the bilinear hybrid system (37).

We formulate now a more general theorem which formulates sufficient conditions of $p$ th mean exponential stabilizability for the stochastic bilinear hybrid system (37) in a case when a single Lyapunov-like function exists. Exemplary simulations are shown in Figures 4 and 5.

Theorem 15. Suppose that the following conditions are satisfied:

(1) there exist symmetric positive definite matrix $\mathbf{H}$, constants $\alpha_{1}^{\mathrm{s}}, \alpha_{1}^{\mathrm{us}}$, and positive constants $\alpha_{2}, \alpha_{3}$ such that

$$
\begin{aligned}
& \bigcup_{l \in \mathcal{S}} \Omega_{l}=\mathscr{R}^{n}, \text { where } \\
& \Omega_{l}^{\text {s }}=\left\{\mathbf{x} \in \mathscr{R}^{n}: \mathbf{x}^{T} \mathbf{H A}(l) \mathbf{x}+\frac{1}{2} \sum_{k=1}^{M} \mathbf{x}^{T} \mathbf{B}_{k}^{T}(l) \mathbf{H B}_{k}(l) \mathbf{x}\right. \\
& \leq \alpha_{1}^{\mathrm{s}} \mathbf{x}^{T} \mathbf{H} \mathbf{x}, \alpha_{1}^{\mathrm{s}}<\left(\alpha_{2}\right)^{2} \wedge \alpha_{2} \mathbf{x}^{T} \mathbf{H} \mathbf{x} \\
&\left.\leq\left|\mathbf{x}^{T} \mathbf{H} \sum_{k=1}^{M} \mathbf{B}_{k}(l) \mathbf{x}\right| \leq \alpha_{3} \mathbf{x}^{T} \mathbf{H} \mathbf{x}\right\} \quad l \in \mathcal{S}, \\
& \Omega_{l}^{\text {us }}=\left\{\mathbf{x} \in \mathscr{R}^{n}: \alpha_{1}^{\mathrm{s}} \mathbf{x}^{T} \mathbf{H} \mathbf{x}<\mathbf{x}^{T} \mathbf{H} \mathbf{A}(l) \mathbf{x}\right. \\
&+\frac{1}{2} \sum_{k=1}^{M} \mathbf{x}^{T} \mathbf{B}_{k}^{T}(l) \mathbf{H} \mathbf{B}_{k}(l) \mathbf{x} \leq \alpha_{1}^{\text {us }} \mathbf{x}^{T} \mathbf{H} \mathbf{x}, \wedge \alpha_{2} \mathbf{x}^{T} \mathbf{H} \mathbf{x} \\
& \leq\left.\left|\mathbf{x}^{T} \mathbf{H} \sum_{k=1}^{M} \mathbf{B}_{k}(l) \mathbf{x}\right| \leq \alpha_{3} \mathbf{x}^{T} \mathbf{H} \mathbf{x}\right\} \quad l \in \mathcal{S},
\end{aligned}
$$




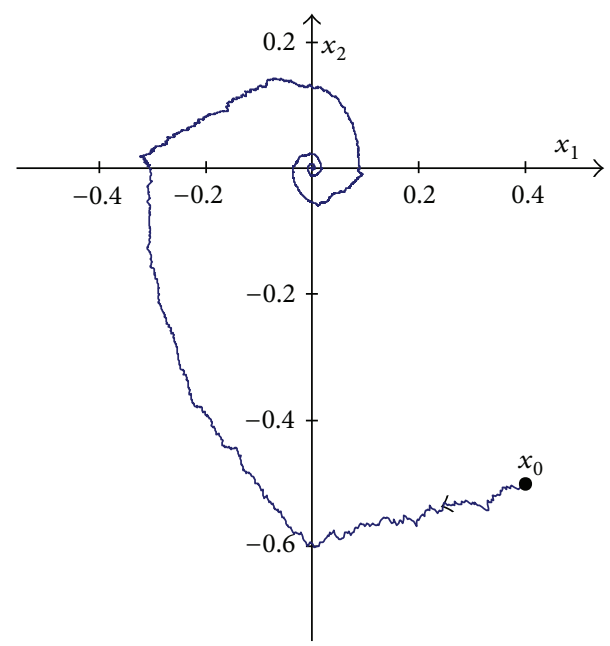

(a) Trajectory of the hybrid system

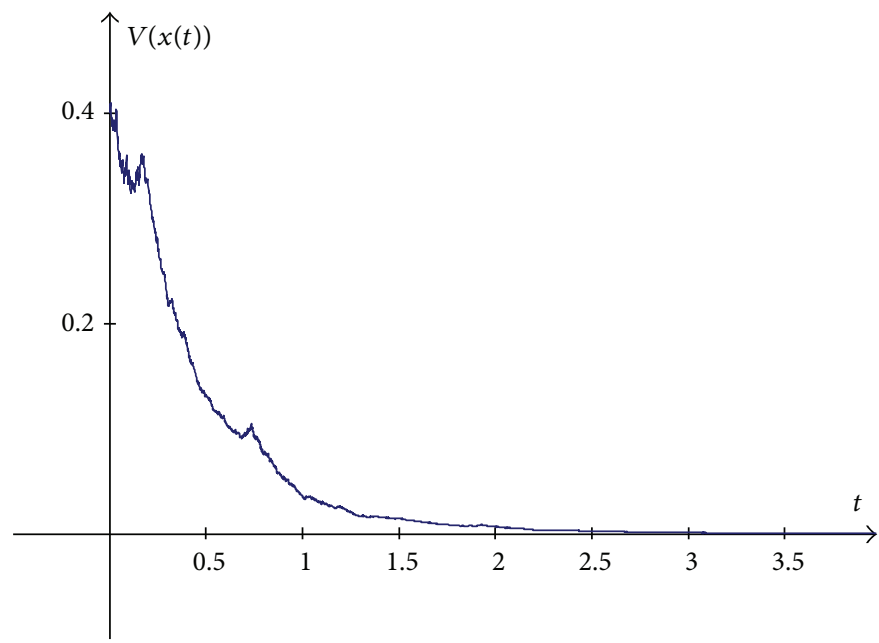

(b) Trajectory of the Lyapunov function

FIGURE 4: Simulation of the hybrid system (53) with stabilizing switching $\sigma=\sigma_{\text {st }}$ for $p=1$.

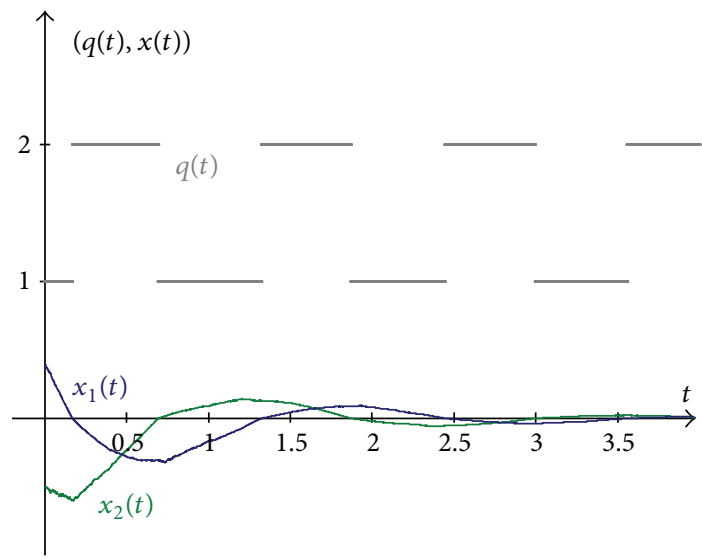

FIGURE 5: Simulation of the hybrid system (53) with stabilizing switching $\sigma=\sigma_{\text {st }}$ for $p=1$.

(2) there exists constant $\kappa>0$ such that $\mathscr{T} \neq \emptyset$, where

$$
\begin{gathered}
\mathscr{T}=\left\{\sigma \in \mathcal{S} \mathscr{W}: \frac{\mathbb{T}_{\mathrm{us}}}{\mathbb{T}_{\mathrm{s}}} \leq \frac{\kappa_{\mathrm{s}}-\kappa}{\kappa_{\mathrm{us}}+\kappa}\right\}, \\
\kappa_{\mathrm{s}}= \begin{cases}p\left(\left|\alpha_{1}^{\mathrm{s}}\right|-\left(\frac{p}{2}-1\right) \alpha_{3}^{2}\right) & \text { dla } 2 \leq p<2+2 \frac{\left|\alpha_{1}^{\mathrm{s}}\right|}{\left(\alpha_{3}\right)^{2}}, \\
p\left(-\alpha_{1}^{\mathrm{s}}-\left(\frac{p}{2}-1\right) \alpha_{2}^{2}\right) & \text { dla } 0<p<2-2 \frac{\alpha_{1}^{\mathrm{s}}}{\left(\alpha_{2}\right)^{2}},\end{cases} \\
\kappa_{\mathrm{us}}=\left\{\begin{array}{rr}
p \leq \alpha_{1}^{\mathrm{s}}<\left(\alpha_{2}\right)^{2}, \\
p\left(\alpha_{1}^{\mathrm{us}}+\left(\frac{p}{2}-1\right) \alpha_{3}^{2}\right) & \text { dla } p \geq 2, \alpha_{1}^{\mathrm{us}} \geq 0, \\
\left.p\left(\frac{p}{2}-1\right) \alpha_{2}^{2}\right) & \text { dla } 0<p<2, \alpha_{1}^{\mathrm{us}} \geq\left(\alpha_{2}\right)^{2} .
\end{array}\right.
\end{gathered}
$$

Then the control $\mathbf{u}: \mathscr{R}^{n} \times \mathcal{S} \rightarrow \mathscr{R}^{m}$ of a form

$$
u_{i}(\mathbf{x}, l)=-p\left(\mathbf{x}^{T} \mathbf{H} \mathbf{x}\right)^{(p / 2)-1} \mathbf{x}^{T} C_{i}^{T}(l) \mathbf{H} \mathbf{x}, \quad i=1,2, \ldots, m, l \in \mathcal{S}
$$

together with the stabilizing switching rule $\bar{\sigma}_{\text {st }} \in \mathscr{T}$ makes the hybrid system (37) the pth mean exponentially stable for

(a) $2 \leq p<2+2\left|\alpha_{1}^{\mathrm{s}}\right| /\left(\alpha_{3}\right)^{2}$ if $\alpha_{1}^{\mathrm{s}}<0$,

(b) $0<p<2-2 \alpha_{1}^{\mathrm{s}} /\left(\alpha_{2}\right)^{2}$ if $0 \leq \alpha_{1}^{\mathrm{s}}<\alpha_{2}^{2}$.

Proof. The thesis follows from Theorem 11. Let us choose a Lyapunov function as follows:

$$
V(\mathbf{x})=\left(\mathbf{x}^{T} \mathbf{H} \mathbf{x}\right)^{(p / 2)}, \quad p>0 .
$$

Function $V$ satisfies assumption (1) of Theorem 11 for $C_{\min }=$ $\lambda_{\min }^{(p / 2)}(\mathbf{H})$ and $C_{\max }=\lambda_{\max }^{(p / 2)}(\mathbf{H})$.

Furthermore,

$$
L_{l} V(\mathbf{x}) \leq \begin{cases}-\kappa_{\mathrm{s}} V(\mathbf{x}), & \text { for } \mathbf{x} \in \Omega_{l}^{\mathrm{s}}, \\ \kappa_{\mathrm{us}} V(\mathbf{x}), & \text { for } \mathbf{x} \in \Omega_{l}^{\mathrm{us}} .\end{cases}
$$

Since condition (51) is satisfied, assumption (2) of Theorem 11 also holds. Assumption (3) of Theorem 11 follows directly. Now using Theorem 11, we obtain that control u : $\mathscr{R}^{n} \times \mathcal{S} \rightarrow$ $\mathscr{R}^{m}$ can be chosen as follows:

$$
\begin{array}{r}
u_{i}(\mathbf{x}, l)=-L_{l}^{i} V(\mathbf{x})=-p\left(\mathbf{x}^{T} \mathbf{H} \mathbf{x}\right)^{(p / 2)-1} \mathbf{x}^{T} \mathbf{C}_{i}^{T}(l) \mathbf{H} \mathbf{x}, \\
i=1,2, \ldots, m, l \in \mathcal{S} .
\end{array}
$$

Hence, the thesis follows.

Notice that function $V(\mathbf{x})=\left(\mathbf{x}^{T} \mathbf{H x}\right)^{(p / 2)}$ is a single Lyapunov-like function for the hybrid system (37). See [16] for the details of the proof. 
TABLE 1: The relationship between the theorems proposed in the paper.

\begin{tabular}{lccc}
\hline & $\begin{array}{c}p \text {-stability of non-linear hybrid } \\
\text { systems }\end{array}$ & $\begin{array}{c}p \text {-stabilizability of non-linear } \\
\text { hybrid systems }\end{array}$ & $\begin{array}{c}p \text {-stabilizability of bilinear } \\
\text { hybrid systems }\end{array}$ \\
\hline $\begin{array}{l}\text { Single Lyapunov function } \\
\text { Single Lyapunov-like function }\end{array}$ & Theorem 6 & $\Leftarrow$ Theorem $10 \Rightarrow$ & Theorem 12 \\
\hline
\end{tabular}

Example 16. Let us consider a special case of the hybrid system (37) given as follows:

$$
\begin{gathered}
d \mathbf{x}(t)=\left[\mathbf{A}(\sigma(t)) \mathbf{x}(t)+u_{1}(\sigma(t), \mathbf{x}(t)) \mathbf{C}_{1}(\sigma(t)) \mathbf{x}(t)\right. \\
\left.+u_{2}(\sigma(t), \mathbf{x}(t)) \mathbf{C}_{2}(\sigma(t)) \mathbf{x}(t)\right] d t \\
+\mathbf{B}(\sigma(t)) \mathbf{x}(t) d w(t), \\
(\sigma(0), \mathbf{x}(0))=\left(\sigma_{0}, \mathbf{x}_{0}\right),
\end{gathered}
$$

where

$$
\begin{gathered}
\mathbf{A}(1)=\left[\begin{array}{cc}
-0.02 & 4 \\
-2 & -0.02
\end{array}\right], \quad \mathbf{C}_{1}(1)=\left[\begin{array}{cc}
0 & 2 \\
-1 & 0
\end{array}\right], \\
\mathbf{C}_{2}(1)=\left[\begin{array}{cc}
1 & 0 \\
-2 & 0
\end{array}\right], \quad \mathbf{B}(1)=\left[\begin{array}{cc}
0.2 & 0 \\
0 & 0.2
\end{array}\right], \\
\mathbf{A}(2)=\left[\begin{array}{cc}
-0.005 & 2 \\
-4 & -0.005
\end{array}\right], \quad \mathbf{C}_{1}(2)=\left[\begin{array}{cc}
-1 & 0 \\
0 & 1
\end{array}\right], \\
\mathbf{C}_{2}(2)=\left[\begin{array}{ll}
0 & 0 \\
0 & 1
\end{array}\right], \quad \mathbf{B}(2)=\left[\begin{array}{cc}
0.1 & 0 \\
0 & 0.1
\end{array}\right] .
\end{gathered}
$$

We study the problem of the $p$ th mean exponential stabilizability for the hybrid system (53) for $p \in(0,2)$. We look for a control vector of a form

$$
\mathbf{u}: \mathcal{S} \times \mathscr{R}^{2} \rightarrow \mathscr{R}^{2}, \quad \mathbf{u}=\left[u_{1}, u_{2}\right]^{T} .
$$

Let us choose the Lyapunov function of a form $V(\mathbf{x})=\mathbf{x}^{T} \mathbf{x}$. Then regions $\Omega_{l}, l \in \mathcal{S}$, are given as follows:

$$
\begin{aligned}
& \Omega_{1}=\left\{\mathbf{x} \in \mathscr{R}^{2}: \mathbf{x}^{T}\left[\mathbf{A}(1)+\frac{1}{2} \mathbf{B}^{T}(1) \mathbf{B}(1)\right] \mathbf{x} \leq 0\right\} \\
&=\left\{\mathbf{x} \in \mathscr{R}^{2}: x_{1} x_{2} \leq 0\right\}, \\
& \Omega_{2}=\left\{\mathbf{x} \in \mathscr{R}^{2}: \mathbf{x}^{T}\left[\mathbf{A}(2)+\frac{1}{2} \mathbf{B}^{T}(2) \mathbf{B}(2)\right] \mathbf{x} \leq 0\right\} \\
&=\left\{\mathbf{x} \in \mathscr{R}^{2}: x_{1} x_{2} \geq 0\right\} \\
& \Omega_{1} \cup \Omega_{2}=\mathscr{R}^{2} .
\end{aligned}
$$

Condition (39) of Theorem 12 is satisfied for $\alpha_{1}=0$. Function $V(\mathbf{x})=\mathbf{x}^{T} \mathbf{x}$ is a single Lyapunov function for the system (53). From Theorem 12, it follows that the control

$$
\begin{gathered}
\mathbf{u}(1, \mathbf{x})=\left[-p\left(\mathbf{x}^{T} \mathbf{H} \mathbf{x}\right)^{(p / 2)-1} x_{1} x_{2},\right. \\
\left.-p\left(\mathbf{x}^{T} \mathbf{H} \mathbf{x}\right)^{(p / 2)-1}\left(x_{1}^{2}-2 x_{1} x_{2}\right)\right] \\
\mathbf{u}(2, \mathbf{x})=\left[-p\left(\mathbf{x}^{T} \mathbf{H} \mathbf{x}\right)^{(p / 2)-1}\left(x_{2}^{2}-x_{1}^{2}\right),-p\left(\mathbf{x}^{T} \mathbf{H} \mathbf{x}\right)^{(p / 2)-1} x_{2}^{2}\right]
\end{gathered}
$$

together with stabilizing switching rule $\sigma_{\text {st }}$ given by

$$
\sigma_{\text {st }}(t)= \begin{cases}1 & \operatorname{gdy} \mathbf{x}(t) \in \Omega_{1}, \\ 2 & \text { gdy } \mathbf{x}(t) \in \Omega_{2},\end{cases}
$$

exponentially $p$ th mean, stabilizes the system (53) for $p \in$ $(0,2)$.

\section{Conclusions}

In this paper, nonlinear and the bilinear hybrid systems parametrically excited by a white noise, consisted of unstable and stable subsystems the described by the Ito stochastic differential equations, have been analyzed. To find sufficient conditions for the $p$ th mean exponential stability and stabilizability, the Lyapunov function techniques and the hybrid control theory have been used. We have found the control of a feedback form, which is a generalization of a feedback control proposed by Florchinger for the nonhybrid systems [11, 12], and the stabilizing switching rule, which is constructed on the basis of the knowledge of the regions of decreasing of the Lyapunov functions for subsystems.

Results for the asymptotic stabilizability under a feedback control of the stochastic nonlinear and bilinear hybrid systems with Markovian or any switching rule have been discussed in [14] and for the $p$ th mean exponential stability and stabilizability of the stochastic linear hybrid system in [4, 16]. The obtained results have been illustrated by an example.

The proposed criteria of the $p$ th mean exponentially stability and stabilizability can be generalized to the hybrid systems parametrically excited by Gaussian colored and nonGaussian noises.

The presented results cannot be compared because they relate to different systems, class. For some class of systems stability conditions obtained using single Lyapunov function approach are the same as those obtained using single Lyapunov-like function approach, while for a wide class of systems, for which Lyapunov-like functions can be used, generally we cannot use single Lyapunov functions. The relationship between the obtained results can be summarized in Table 1.

\section{Acknowledgment}

The authors gratefully acknowledge research support from Cardinal Stefan Wyszyński University in Warsaw.

\section{References}

[1] D. Liberzon, Switching in Systems and Control, Birkhäauser, Boston, Mass, USA, 2003. 
[2] D. Liberzon and A. S. Morse, "Basic problems in stability and design of switched systems," IEEE Control Systems Magazine, vol. 19, pp. 59-70, 1999.

[3] M. Wang and J. Zhao, "Quadratic stabilization of a class of switched nonlinear systems via single Lyapunov function," Nonlinear Analysis, vol. 4, no. 1, pp. 44-53, 2010.

[4] E. Seroka and L. Socha, "Some remarks on stabilizability of linear stochastic hybrid systems," in Proceedings of the 18th World Congress of the International Federation of Automatic Control (IFAC) World Congress, vol. 18, pp. 5724-5729, Milano, Italy, 2011.

[5] J. Raouf and H. Michalska, "Stabilization of switched linear systems with Wiener process disturbances," in Proceedings of the American Control Conference, pp. 3281-33286, Baltimore, Md, USA, July 2010.

[6] S. Pettersson and B. Lennartson, "Hybrid system stability and robustness verification using linear matrix inequalities," International Journal of Control, vol. 75, no. 16-17, pp. 1335-1355, 2002, Switched, piecewise and polytopic linear systems.

[7] N. H. El-Farra, P. Mhaskar, and P. D. Christofides, "Output feedback control of switched nonlinear systems using multiple Lyapunov functions," Systems and Control Letters, vol. 54, no. 12, pp. 1163-1182, 2005.

[8] G. Zhai, B. Hu, K. Yasuda, and A. N. Michel, "Stability analysis of switched systems with stable and unstable subsystems: an average dwell time approach," International Journal of Systems Science, vol. 32, no. 8, pp. 1055-1061, 2001.

[9] D. V. Dimarogonas and K. J. Kyriakopoulos, "Lyapunov-like stability of switched stochastic systems," in Proceedings of the American Control Conference, pp. 1868-1872, Boston, Mass, USA, June 2004.

[10] H. Lin and P. J. Antsaklis, "Stability and stabilizability of switched linear systems: a survey of recent results," IEEE Transactions on Automatic Control, vol. 54, no. 2, pp. 308-322, 2009.

[11] P. Florchinger, "A stochastic Jurdjevic-Quinn theorem," SIAM Journal on Control and Optimization, vol. 41, no. 1, pp. 83-88, 2002.

[12] P. Florchinger, "A stochastic version of Jurdjevic-Quinn theorem," Stochastic Analysis and Applications, vol. 12, no. 4, pp. 473480, 1994.

[13] A. V. Skorohod, Asymptotic Methods in the Theory of Stochastic Differential Equations, American Mathematical Society, 2004.

[14] E. Seroka and L. Socha, "Stabilizability of a class of stochastic bilinear hybrid systems," Journal of Mathematical Analysis and Applications, vol. 384, no. 2, pp. 658-669, 2011.

[15] X. Mao, Stochastic Differential Equations and Their Applications, Horwood Publishing Limited, Chichester, UK, 1997.

[16] E. Seroka and L. Socha, "Remarks on p-stabilizability of stochastic linear hybrid systems," in Proceedings of the IFAC Conference on Analysis and Design of Hybrid Systems (ADHS '12), pp. 400405, Eindhoven, The Netherlands, 2012. 


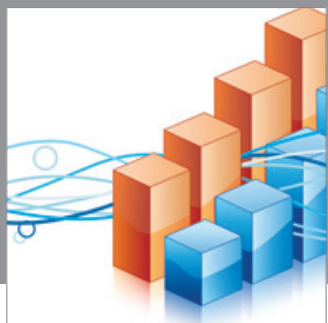

Advances in

Operations Research

mansans

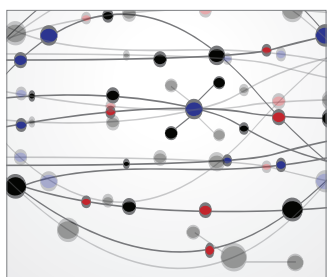

The Scientific World Journal
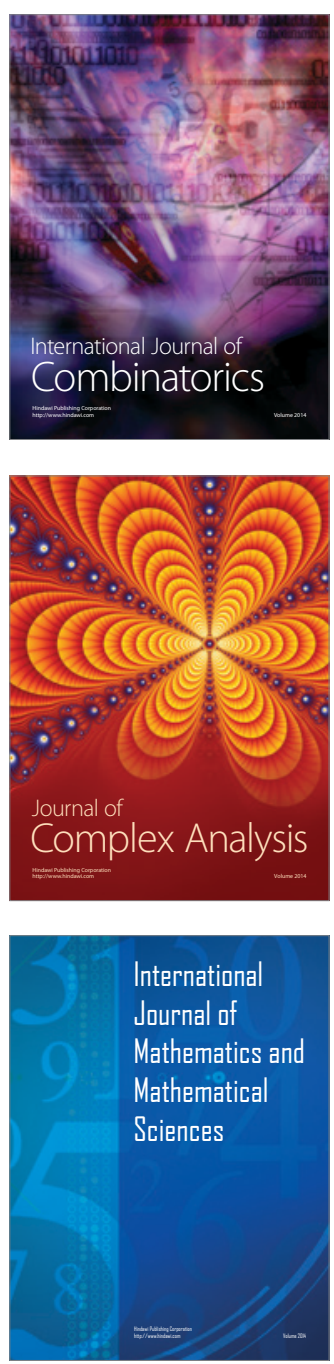
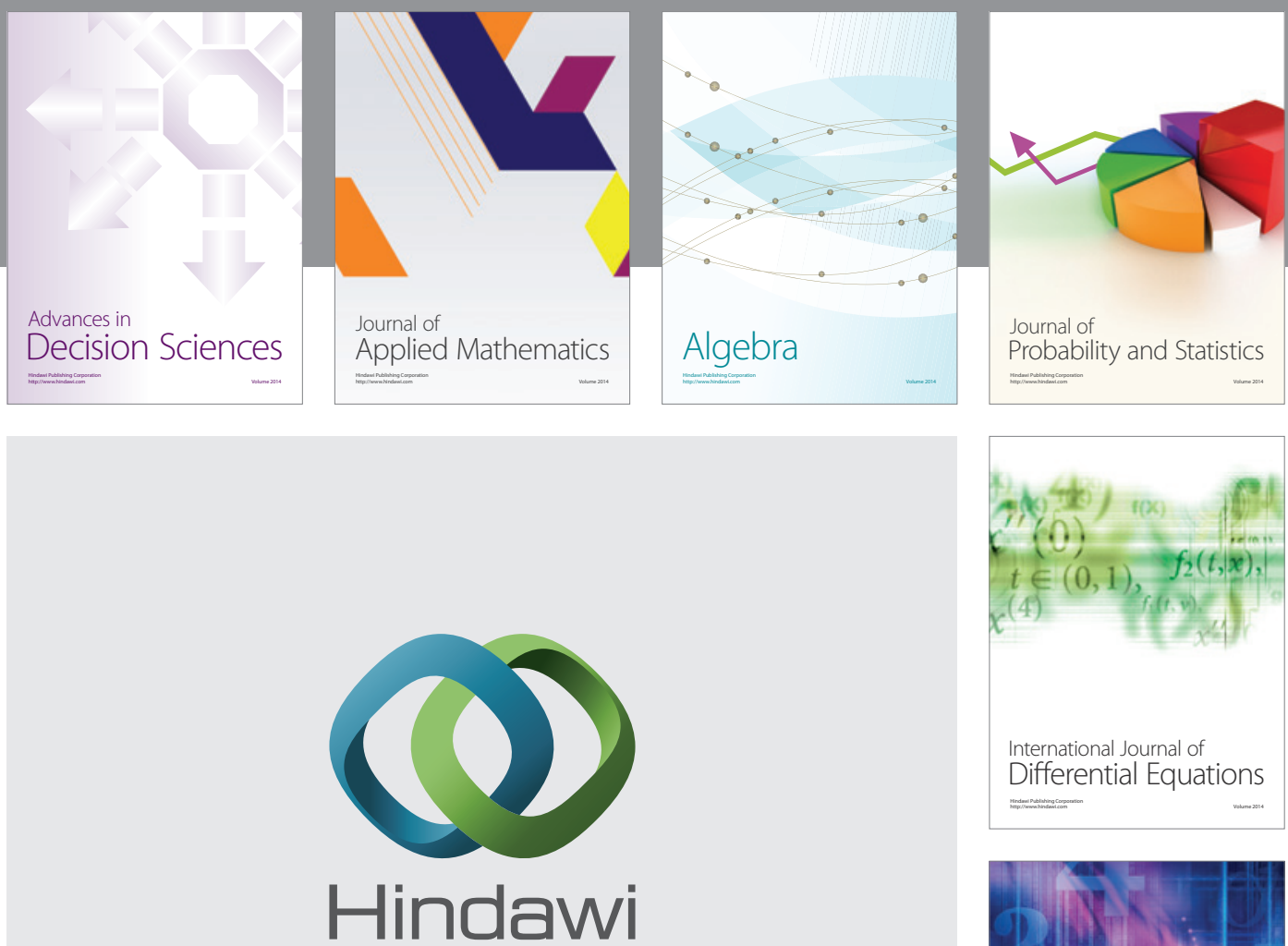

Submit your manuscripts at http://www.hindawi.com
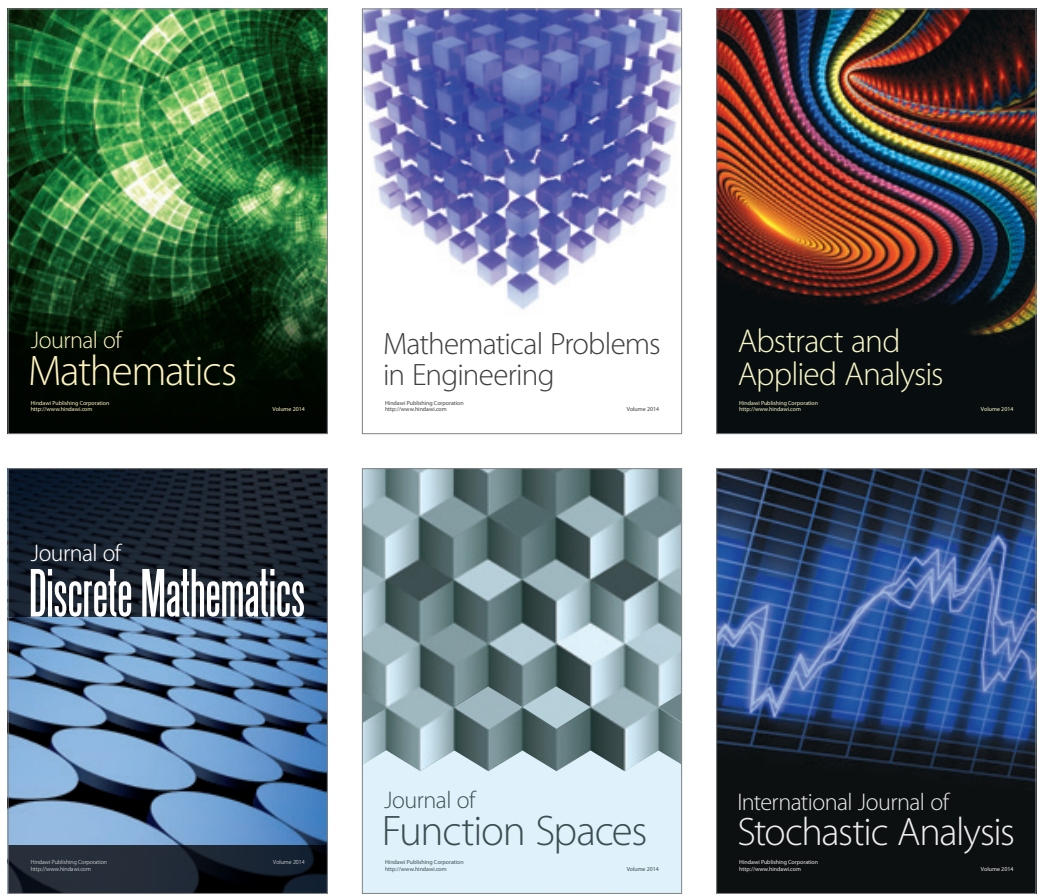

Journal of

Function Spaces

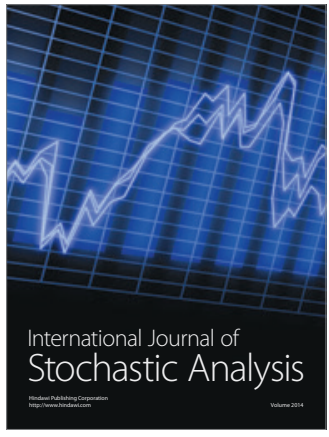

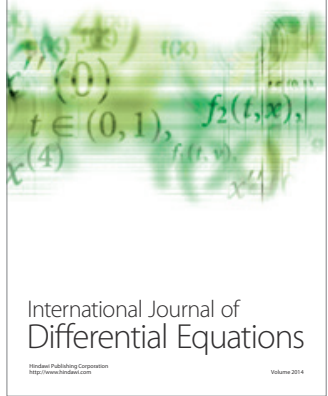
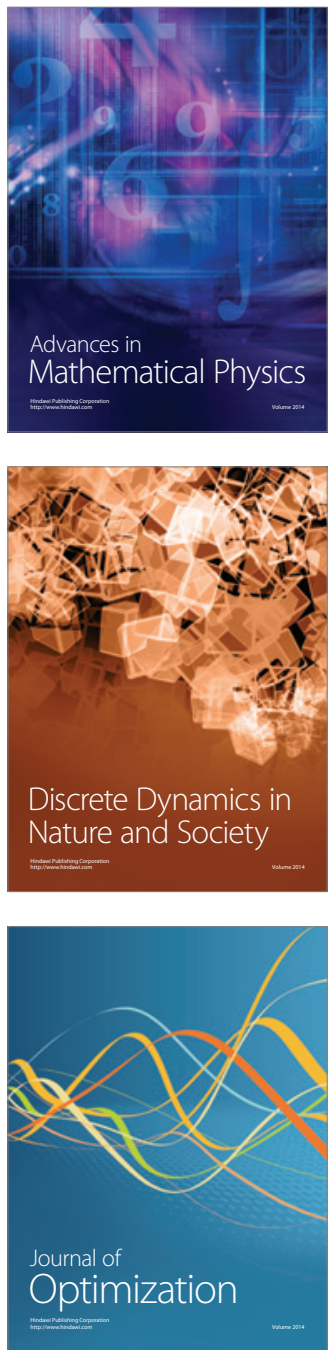